\title{
Myopathic changes in malignant hyperthermia-susceptible patients
}

\author{
Henry Rosenberg, MD
}

Received: 3 July 2013/Accepted: 3 July 2013 / Published online: 30 July 2013

(C) Canadian Anesthesiologists' Society 2013

With each passing year, it becomes more apparent that mutations in the ryanodine receptor gene (RYR1), the gene most associated with susceptibility of malignant hyperthermia $(\mathrm{MH})$, are also associated with a variety of subclinical as well as overt myopathies. This is true for DNA changes proven to be causal for MH (mutations) as well as variants associated with $\mathrm{MH}$ but whose role in the pathophysiology of MH is not firmly established. Several characterized myopathies, such as central core disease, multiminicore disease, and congenital fibre type disproportion, display RYR1 mutations and often predispose to MH susceptibility. Recent studies show that some patients with myalgia and exercise-induced rhabdomyolysis unrelated to known myopathies also harbour RYR1 variants. ${ }^{1}$ Nevertheless, there are only a few studies of the histologic changes in large cohorts of MH-susceptible patients. ${ }^{2}$ Such investigations may be scarce because the focus is on the contracture response to halothane, caffeine, and other agents (and more recently on the genetic changes) when patients are referred for $\mathrm{MH}$ diagnostic testing, and an expert may not perform or interpret an histologic examination. In addition, earlier studies of MH muscle revealed non-specific changes in muscle histology. Over 30 years ago, Drs. Britt and Kalow first established the MH Investigation Unit at the University of Toronto. Dr. David MacLennan has long been associated with the Unit, and more recently, it has

H. Rosenberg, MD

Malignant Hyperthermia Association of the USA,

Sherburne, NY, USA

H. Rosenberg, MD ( $₫)$

Department of Medical Education and Clinical Research,

Saint Barnabas Medical Center, Livingston, NJ, USA

e-mail: henryrosenberg@yahoo.com been headed by Dr. Sheila Riazi. It is fortunate that the Investigation Unit has collected and analyzed muscle for histologic changes, performed contracture testing, and implemented molecular genetic analysis when that became available. These data formed the basis for Orlov et al. $\mathrm{s}^{3}$ comprehensive review of the histomorphology of 399 MH-susceptible patients diagnosed by caffeine/ halothane contracture testing during 1992-2011.

After excluding seven patients with demonstrated typical changes of central core disease, the authors' key finding was that $22 \%$ of $\mathrm{MH}$-susceptible patients were found to have a range of non-specific histologic changes, such as type 2 fibre atrophy, fibre size variability, necrotic fibres, and ragged red fibres. Six patients had severe disruption of the muscle structure associated with muscle pain and cramping. All but one patient (a carrier for Duchenne's muscular dystrophy) were probands harbouring $\mathrm{MH}$-causative mutations. In general, probands were more likely to exhibit anatomic changes than patients who were tested for MH susceptibility for other reasons. In order to ensure that the histologic changes were not a result of the MH episode, at least six months elapsed from episode to muscle biopsy.

Findings in this study are similar to other studies in the MH-susceptible population. For example, a study of genotype, phenotype, and histologic changes in $175 \mathrm{MH}-$ susceptible families from European $\mathrm{MH}$ testing centres found that about $20 \%$ of patients were found to have histologic changes, primarily "cores" without overt signs of central core disease. ${ }^{2}$ In that study, $60 \%$ of patients displayed an RYR1 variant, and 58 of 179 susceptible patients displayed a causative mutation. In the present study by Orlov et al., 57\% of patients displayed an RYR1 variant, and an MH causative mutation was identified in 86 of the 392 (22\%) susceptible patients. 
Similarly, in a recent study from the North American MH Registry of the Malignant Hyperthermia Association of the United States, 52\% of $120 \mathrm{MH}$-susceptible patients were found to have an RYR1 variant, and $21 \%$ harboured a causal MH mutation. ${ }^{4}$ It is also of interest that three of the most frequent causal mutations found in that study and three of the most common causal mutations found in the study by Monnier $^{2}$ are among the five most frequent mutations reported in the study by Orlov et al. Based on the very stringent criteria required to prove causality, it is yet unknown how many of the variants not yet characterized as being causal for $\mathrm{MH}$ are actually pathogenic. One of the great unanswered questions related to RYR1 myopathies is the functional significance of the DNA changes in this large gene. In the case of $\mathrm{MH}$, the relevant question is which variants are causally related to clinical manifestations of MH. It appears, therefore, that multiple studies point to similar findings related to abnormal histology and prevalence of DNA variants in RYR1. As newer more robust sequencing techniques (next-generation sequencing) are applied to the RYR1 gene and additional functional studies are performed on variants, it is likely that $70-90 \%$ of susceptible patients will display one or more causal mutations. Only one other gene, the dihydropyridine gene (CACNA1S), has been shown to harbour $\mathrm{MH}$ causative mutations in about $1 \%$ of the $\mathrm{MH}$ population.

When we turn to the issue of clinical myopathic findings in patients with RYR1 DNA variants, several studies are relevant.

Children suffering from congenital myopathies regularly display DNA changes in the RYR1 gene. Pediatric neurologists consider ryanodine receptor mutations as the commonest cause of congenital myopathies. ${ }^{5}$

Results of a very recent multicentre study in adults showed that $35 \%$ of 39 unrelated families harbour one or more RYR1 mutations where one or more individuals have experienced rhabdomyolysis and/or exertional myalgia (without anesthesia). ${ }^{1}$ Individual case reports and small series of dramatic instances of rhabdomyolysis and myalgia have similarly identified an association with RYR1 mutations. In one large family, a young male with unexplained muscle weakness and elevated creatine kinase (CK) levels was determined to be $\mathrm{MH}$ susceptible by contracture testing. Seventeen of his relatives shared a common RYR1 mutation (p.Thr2206Met) and also manifested histopathologic changes ranging from multiminicores to "moth-eaten fibres". ${ }^{6}$ In the era before genetic testing, studies showed that about $50 \%$ of patients with unexplained elevated CK were also MH susceptible on contracture testing. ${ }^{7}$ It has been even more dramatic to encounter the occasional case of sudden death from an MH-like event associated with mutations in the RYR1 gene. ${ }^{8}$
These as well as other studies clearly point to an association between mutations in RYR1, MH susceptibility, and a variety of subclinical and sometimes clinically apparent myopathy. In the study by Orlov et al. as well as other studies, it is important to point out that no difference in the prevalence of RYR1 causative mutations as a function of abnormal histology has been found. In other words, it is not possible to relate the severity of expression of $\mathrm{MH}$, either clinically or histologically, to a particular mutation in the RYR1 gene. This is not surprising since it is now well known that numerous factors influence gene expression.

Perhaps the association between MH susceptibility and the prevalence of adult myopathy has been underappreciated because most $\mathrm{MH}$-susceptible individuals and their families have not been followed for years after their MH episode. Therefore, a late onset of non-specific signs, such as mild muscle weakness, muscle cramping, and pain, could be overlooked.

The fact that muscles in more than $20 \%$ of $\mathrm{MH}-$ susceptible individuals have abnormal histomorphology and a subgroup displays signs of frank myopathy, microscopically as well as clinically, indicates that it may be time to reframe our view of MH. Rather than consider $\mathrm{MH}$ as an episodic metabolic response to certain volatile anesthetics and succinylcholine and rarely environmental factors, MH should be thought of as a myopathy that places some patients at risk for a variety of muscle-related signs, including mild to severe muscle pain, significant muscle weakness (e.g., patients with central core disease), rhabdomyolysis, classic $\mathrm{MH}$ response to anesthetics, and MH-like episodes unrelated to anesthesia.

Since Orlov et al.'s study shows that probands are more likely to reveal histomorphologic changes than the general MH-susceptible population, it would seem appropriate that confirmation of $\mathrm{MH}$ susceptibility by either contracture testing or molecular genetic testing should also include a thorough neuromuscular exam and appropriate laboratory studies, including histology in those patients This may seem self evident, but only a limited number of patients suspected of having experienced an $\mathrm{MH}$ episode are referred for confirmatory testing and analysis. Based on current data, it is impossible to ascertain the number of patients suspected of experiencing an $\mathrm{MH}$ episode who undergo confirmatory testing. Factors such as cost of testing, difficulty in interpretation of test results, and lack of knowledge of the implications of $\mathrm{MH}$ by general physicians and neurologists often limit such follow up.

In conclusion, the current study confirms that muscle histology per se is not very useful as a sole diagnostic test for MH susceptibility; however, histology can be most informative in patients who experienced an $\mathrm{MH}$ episode. The study also shows that the spectrum of clinical 
manifestations of mutations and DNA variants of the RYR1 gene is varied and extensive. Future studies of phenotype (both clinical and histologic) and genotype will hopefully serve to identify those at risk for the most clinically significant manifestations of $\mathrm{MH}$ and other ryanodinopathies.

The MH Investigation Unit at the University of Toronto has been and continues to be a source of important information concerning $\mathrm{MH}$ and $\mathrm{MH}$-like syndromes.

\section{Les modifications myopathiques chez les patients susceptibles à l'hyperthermie maligne}

Au fil des années, il devient de plus en plus évident que des mutations au niveau du gène du récepteur de la ryanodine (RYR1), le gène le plus communément associé à la susceptibilité à l'hyperthermie maligne (HM), sont également associées à d'autres myopathies, qu'elles soient asymptomatiques ou non. Cela est vrai pour les modifications de l'ADN pour lesquelles il a été démontré qu'elles jouaient un rôle causal dans l'HM (mutations) aussi bien que pour les variantes associées à l'HM mais dont le rôle dans la physiopathologie de cette affection n'est pas clairement déterminé. Plusieurs myopathies caractérisées, comme par exemple la myopathie congénitale à axe central, la myopathie à multi-minicores et la myopathie par disproportion des fibres musculaires, présentent des mutations du RYR1 et prédisposent souvent à une susceptibilité à l'HM. Des études récentes ont montré que certains patients atteints de myalgie et de rhabdomyolyse induite par l'exercice, non liées à des myopathies connues, possèdent également des variantes du RYR1. ${ }^{1}$ Néanmoins, il n'existe que quelques études s'intéressant aux modifications histologiques chez d'importantes cohortes de patients susceptibles à l'HM. ${ }^{2}$ La raison de ce manque de données pourrait être liée au fait qu'on porte principalement attention à la réaction de contracture à l'halothane, à la caféine et à d'autres agents (et plus récemment aux changements génétiques) lorsque des patients sont référés pour subir des tests diagnostiques d'HM; par conséquent, un expert pourrait ne pas réaliser ou interpréter d'examen histologique. En outre, des études anciennes sur des muscles de patients atteints d'HM ont révélé des changements non spécifiques de l'histologie musculaire. Il y a plus de 30 ans, les Drs Britt et Kalow fondaient l'Unité de recherche sur l'HM à l'Université de Toronto. Le Dr David MacLennan a longtemps été associé à cette unité et, plus récemment, la Dre Sheila Riazi en a pris la tête. Fait heureux, l'Unité de recherche a colligé et analysé les changements histologiques dans ces muscles, réalisé des tests de contracture, et implanté l'analyse génétique moléculaire lorsque celle-ci est devenue disponible. Ces données sont à la base du compte-rendu exhaustif d'Orlov et coll. sur l'histomorphologie de 399 patients susceptibles à l'HM diagnostiqués grâce au test de contracture à la caféine et à l'halothane entre 1992 et 2011.

Après avoir exclu sept patients qui démontraient des modifications typiques de myopathie congénitale à axe central, la découverte principale des auteurs est que $22 \%$ des patients susceptibles à l'HM manifestaient divers changements histologiques non spécifiques, tels qu'une atrophie des fibres musculaires de type 2 , une variabilité de la taille des fibres, des fibres nécrotiques et des fibres rouges déchiquetées. Six patients présentaient une désorganisation grave de la structure musculaire associée à des douleurs musculaires et des crampes. À l'exception d'un patient (porteur d'une dystrophie musculaire de Duchenne), tous les patients étaient des proposants possédant des mutations causant l'HM. En général, les proposants étaient plus susceptibles de manifester des changements anatomiques que les patients testés pour une susceptibilité à l'HM pour d'autres raisons. Afin de s'assurer que les changements histologiques n'étaient pas le résultat de l'épisode d'HM, au moins six mois se sont écoulés entre l'épisode et la biopsie musculaire.

Les observations tirées de cette étude rejoignent celles d'autres études portant sur la population susceptible à l'HM. Par exemple, une étude du génotype, du phénotype et des changements histologiques de 175 familles susceptibles à l'HM dans les centres de test de l'HM en Europe a révélé qu'environ $20 \%$ des patients présentaient des changements histologiques, principalement des « cores » sans signes patents de myopathie congénitale à axe central. ${ }^{2}$ Dans cette étude, $60 \%$ des patients possédaient une variante du RYR1, et 58 des 179 patients susceptibles avaient une mutation causative. Dans l'étude d'Orlov et coll. présentée ici, $57 \%$ des patients possédaient une variante du RYR1, et une mutation causative d'HM a été identifiée chez 86 des 392 (22\%) patients susceptibles.

De même, dans une étude récente du Registre nord-américain d'HM (North American MH Registry) de l'Association américaine de l'hyperthermie maligne (Malignant Hyperthermia Association of the United States), on a observé que $52 \%$ des 120 patients susceptibles à l'HM possédaient une variante du RYR1, et $21 \%$ une mutation causale d'HM. ${ }^{4}$ Il est également intéressant que trois des mutations causales les plus fréquentes observées dans cette étude et trois des mutations causales les plus fréquentes observées dans l'étude de Monnier ${ }^{2}$ se trouvent parmi les cinq mutations 
les plus fréquentes rapportées dans l'étude d'Orlov et coll. Sur la base des critères extrêmement rigoureux requis pour attester un lien de causalité, nous ne savons pas encore dans quelle mesure plusieurs des variantes non encore caractérisées comme étant causales d'HM sont en fait pathogènes. L'une des grandes questions sans réponse liée aux myopathies du RYR1 est la signification fonctionnelle des changements de l'ADN dans ce gène de taille importante. Dans le cas de l'HM, la question pertinente est de savoir quelles variantes ont une relation causale avec les manifestations cliniques de l'HM. Ainsi, il semble que plusieurs études pointent vers des résultats semblables liés à une histologie anormale et à la prévalence de variantes de l'ADN dans le RYR1. Lorsque de nouvelles techniques de séquençage plus robustes (le séquençage de nouvelle génération) seront appliquées au gène RYR1 et que d'autres études fonctionnelles seront réalisées sur ses variantes, il est probable que $70-90 \%$ des patients susceptibles présenteront au moins une mutation causale. On a trouvé un autre gène, le gène du récepteur de la dihydropyridine (CACNA1S), qui possédait des mutations causatives d'HM chez environ $1 \%$ de la population atteinte d'HM.

Lorsqu' on se tourne vers la question des observations cliniques de myopathie chez les patients possédant des variantes de l'ADN du RYR1, plusieurs études sont pertinentes.

Les enfants atteints de myopathies congénitales présentent souvent des mutations de l'ADN du gène RYR1. Les neurologues pédiatres considèrent les mutations du récepteur de la ryanodine comme la cause la plus courante de myopathies congénitales. ${ }^{5}$

Les résultats d'une étude multicentrique très récente chez des adultes montrent que $35 \%$ de 39 familles sans liens de sang avaient au moins une mutation du RYR1 lorsqu'un ou plusieurs de leurs membres avait souffert de rhabdomyolyse et/ou de myalgie induite par l'exercice (sans anesthésie). ${ }^{1}$ Des présentations de cas individuels et de petites séries d'exemples dramatiques de rhabdomyolyse et de myalgie ont également démontré une association avec des mutations du RYR1. Dans une grande famille, on a découvert grâce à un test de contracture qu'un jeune homme présentant une faiblesse musculaire inexpliquée et des taux de créatine kinase élevés était susceptible à l'HM. Dix-sept membres de sa famille partageaient une mutation commune du RYR1 (p.Thr2206Met) et manifestaient également des changements histopathologiques allant de multi-minicores à des «fibres mitées ». ${ }^{6}$ À l'époque précédant les tests génétiques, des études ont montré qu'environ $50 \%$ des patients présentant des niveaux de CK élevés inexpliqués étaient également susceptibles à l'HM selon le test de contracture. ${ }^{7}$ On a même rencontré, de façon occasionnelle, le cas d'une mort soudaine causée par un épisode semblable à l'HM et associé à des mutations du gène RYR $1 .{ }^{8}$

Ces études, tout comme d'autres, indiquent clairement une association entre les mutations du RYR1, une susceptibilité à l'HM, et une diversité de myopathies frustres et parfois apparentes d'un point de vue clinique. Dans l'étude d'Orlov et coll. ainsi que dans d'autres, il est important de souligner qu'on n'a observé aucune différence dans la prévalence de mutations causatives du RYR1, que l'histologie soit normale ou non. Autrement dit, il n'est pas possible de lier la gravité de l'expression de l'HM, de façon clinique ou histologique, à une mutation particulière du gène RYR1. Cela n'est pas surprenant étant donné qu'il est aujourd'hui bien établi que de nombreux facteurs influencent l'expression génétique.

Peut-être l'association entre la susceptibilité à l'HM et la prévalence de la myopathie chez l'adulte a-t-elle été sous-estimée parce que la plupart des personnes susceptibles à l'HM et leurs familles n'ont pas été suivies au cours des années suivant l'épisode d'HM. Ainsi, l'apparition tardive de signes non spécifiques, tels qu'une légère faiblesse musculaire, des crampes musculaires et des douleurs, pourrait ne pas avoir été pris en compte.

Le fait que les muscles de plus de $20 \%$ des personnes susceptibles à l'HM présentent une histomorphologie anormale et qu'un sous-groupe montre des signes à la fois microscopiques et cliniques de myopathie franche indique qu'il est peut-être temps de repenser notre conception de l'HM. Plutôt que d'envisager l'HM comme une réponse métabolique épisodique à certains anesthésiques volatils, à la succinylcholine et, rarement, à des facteurs environnementaux, l'HM devrait être considérée comme une myopathie qui met certains patients à risque de symptomatologie musculaire, y compris de douleurs musculaires légères à graves, d'une faiblesse musculaire importante (par ex., les patients atteints de myopathie congénitale à axe central), de rhabdomyolyse, de réponse d'HM classique aux anesthésiques, et d'épisodes semblables à l'HM mais n'ayant rien à voir avec une anesthésie.

Étant donné que l'étude d'Orlov et coll. montre que les proposants sont plus enclins à révéler des changements histomorphologiques que la population susceptible à l'HM en général, il semble logique qu'une confirmation de la susceptibilité à l'HM par test de contracture ou par test génétique moléculaire comprenne également un examen neuromusculaire complet et des tests de laboratoire adaptés, y compris une histologie chez ces patients. Cela pourrait paraître évident, mais seul un nombre restreint de patients qu'on soupçonne d'avoir subi un épisode d'HM sont référés pour passer des tests de confirmation du diagnostic et des analyses. En nous fondant sur les données 
disponibles à l'heure actuelle, il est impossible de déterminer précisément le nombre de patients qu'on soupçonne avoir vécu un épisode d'HM et qui subissent des tests pour confirmer ces soupçons. Des facteurs tels que le coût des tests, la difficulté d'interprétation des résultats et le manque de connaissance des implications de l'HM par les omnipraticiens et les neurologues limitent souvent un tel suivi.

Pour conclure, l'étude présentée ici confirme que l'histologie musculaire n'est pas, en soi, très utile comme test diagnostique unique de la susceptibilité à l'HM; toutefois, l'histologie peut fournir une foule de renseignements à propos des patients ayant subi un épisode d'HM. Cette étude montre également que l'éventail des manifestations cliniques des mutations et des variantes ADN du gène RYR1 est vaste et varié. Des études futures du phénotype (cliniques et histologiques) et du génotype serviront, nous l'espérons, à identifier les personnes courant le risque de subir les manifestations les plus importantes d'un point de vue clinique de l'HM et d'autres myopathies liées à la ryanodine.

L'Unité de recherche sur l'HM de l'Université de Toronto a été et continue d'être une source d'informations importantes concernant l'HM et les syndromes ressemblant à l'HM.

Conflicts of interest None declared.

\section{References}

1. Dlamini N, Voermans NC, Lillis $S$, et al. Mutations in RYR1 are a common cause of exertional myalgia and rhabdomyolysis. Neuromuscul Disord 2013; 23: 540-8.

2. Monnier N, Kozak-Ribbens G, Krivosic-Horber R, et al. Correlations between genotype and pharmacological, histological, functional, and clinical phenotypes in malignant hyperthermia susceptibility. Human Mutation 2005; 26: 413-25.

3. Orlov D, Keith J, Rosen D, Croul S, Kraeva N, Riazi S. Analysis of histomorphology in malignant hyperthermia-susceptible patients. Can J Anesth 2013; 60: this issue. DOI:10.1007/s12630-013-0005-9.

4. Brandom BW, Bina $S$, Wong $C A$, et al. Ryanodine receptor type 1 gene variants in the malignant hyperthermia-susceptible population of the United States. Anesth Analg 2013; 116: 1078-86.

5. Jungbluth H, Sewry CA, Muntoni F. Core myopathies. Semin Pediatr Neurol 2011; 18: 239-49.

6. Rueffert H, Wehner M, Ogunlade V, Meinecke CD, Schober R. Mild clinical and histopathological features in patients who carry the frequent and causative malignant hyperthermia RYR1 mutation pThr2206Met. Clin Neuropathol 2009; 28: 409-16.

7. Lingaraju $N$, Rosenberg $H$. Unexplained increases in serum creatine kinase levels: its relation to $\mathrm{MH}$ susceptibility. Anesth Analg 1991; 72: 702-5.

8. Lavezzi WA, Capacchione JF, Muldoon SM, et al. Case report: Death in the emergency department: an unrecognized awake malignant hyperthermia-like reaction in a six-year-old. Anesth Analg 2013; 116: 420-3.

Conflit d'intérêt Aucun. 